\title{
Review
}

Interational Archives of
Allergy
Immunology
Int Arch Allergy Immunol 2012;159:1-5

DOI: $10.1159 / 000336374$

Published online: April 27, 2012

\section{Evaluation of Mast Cell Activation Syndromes: Impact of Pathology and Immunohistology}

\author{
H.-P. Horny ${ }^{\mathrm{a}} \quad$ K. Sotlar ${ }^{\mathrm{a}} \quad$ P. Valent ${ }^{\mathrm{b}}$

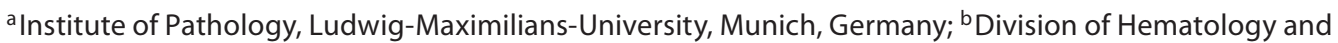 \\ Hemostaseology, Department of Internal Medicine I, Medical University of Vienna, Vienna, Austria
}

\section{Key Words}

Mast cells • Mast cell activation syndromes • Mastocytosis • Pathology $\cdot$ Bone marrow $\cdot$ CD25

\begin{abstract}
Mast cell activation syndromes (MCAS) are clinically defined disease states with a largely unknown morphological background. Since mastocytosis may be associated with MCAS, it is crucial in every patient to document or exclude mastocytosis by appropriate histological, molecular, and serological investigations of tissues/organs that are commonly involved in mastocytosis like skin, mucosa of the gastrointestinal tract and bone marrow. Accordingly, histopathological investigation including immunohistological stains is crucial to reach the final diagnosis in such patients and to classify MCAS into primary MCAS, which can present with or without evidence of overt mastocytosis, or secondary MCAS, where an underlying disease with or without tissue inflammation is detected. Cases without evidence of mastocytosis, monoclonal mast cells, or any underlying disease should be termed idiopathic MCAS. When the activating point mutant KIT D816V is detectable but criteria for diagnosis of mastocytosis are not completely met, a so-called (mono)clonal MCAS as a subvariant of primary MCAS should be diagnosed.
\end{abstract}

Copyright $\odot 2012$ S. Karger AG, Basel

\section{KARGER}

Fax +4161306 1234

E-Mail karger@karger.ch

www.karger.com (c) 2012 S. Karger AG, Basel

$1018-2438 / 12 / 1591-0001 \$ 38.00 / 0$

Accessible online at:

www.karger.com/iaa

\section{Introduction}

Mastocytosis is an extremely heterogeneous disorder of bone marrow origin ranging from self-limiting cutaneous diseases in the childhood to aggressive or leukemic systemic disorders with fatal course in the adult (table 1). Systemic mastocytosis (SM) is defined as histologically proven involvement of at least one extracutaneous tissue and is usually diagnosed in the bone marrow. Histological investigation including immunohistochemical stainings with antibodies against three important mast cell-related antigens, namely tryptase, CD117 (KIT) and CD25, allows a diagnosis of mastocytosis to be established or excluded in almost all cases [1]. While coexpression of tryptase and CD117 defines the normal phenotype of mast cells present in all reactive and neoplastic states, expression of CD25 is seen almost exclusively in SM.

In 2001 and updated in 2008, the WHO defined diagnostic criteria for mastocytosis and its various subtypes $[2,3]$. For SM, the so-called SM criteria are widely used (table 2). However, in a smaller cohort of patients, SM criteria are not met and even if KIT D816V is detectable and the patients are suffering from typical clinical mediatorrelated symptoms a diagnosis of SM cannot be established. For these patients, the preliminary term monoclo- 
Table 1. Classification of mast cell disorders

1 Cutaneous mastocytosis (CM)

2 Indolent systemic mastocytosis (ISM)

a Smoldering systemic mastocytosis (SSM)

b Bone marrow mastocytosis (BMM)

c Well-differentiated ISM (WDISM)

\begin{tabular}{ll}
\hline 3 & Aggressive systemic mastocytosis (ASM) \\
\hline 4 & SM-AHNMD \\
\hline 5 & Mast cell leukemia (MCL) \\
\hline 6 & Mast cell sarcoma (MCS) \\
\hline 7 & Extracutaneous (benign) mastocytoma
\end{tabular}

* Associated non-mast-cell clonal hematological disorder.

A primary MCAS can occur in any type of mast cell disorder.

Table 2. Systemic mastocytosis: diagnostic criteria

Main criterion

Compact infiltrate in an extramedullary organ ( $>15$ mast cells)

Minor criteria

Prominent spindling of mast cells ( $>25 \%$ in a compact infiltrate)

Aberrant immunophenotype of mast cells (CD2 and/or CD25)

Activating point mutation of $c$-kit at codon 816

(usually KIT D816V)

Elevated baseline serum tryptase $(>20 \mathrm{ng} / \mathrm{ml})$

Diagnosis of systemic mastocytosis can be established when the major and at least 1 minor criterion or at least 3 minor criteria are fulfilled.

nal mast cell activation syndrome (mMCAS) has been proposed $[4,5]$.

So far, it remains unknown whether or not MCAS is a pre-phase of mastocytosis, and whether or not also other forms of severe anaphylaxis may be related to an abnormal mast cell activation or even mast cell monoclonality $[6,7]$. To explore these possibilities and to define the impact of pathological staging and grading in these patients, a consensus conference was organized in 2010 in Vienna, Austria. The general outcome of this conference has been presented in a separate publication. In the current article, the impact and value of appropriate histological and immunohistochemical evaluation in patients with clinical signs of unexplained mast cell activation is highlighted, thereby underlining the crucial role of the pathologist for definitive subtyping of MCAS, which can be a primary condition with or without $\mathrm{SN}$, a secondary MCAS, or idiopathic MCAS. It cannot be overemphasized that knowledge of all relevant clinical information is also of major importance to achieve a correct diagnosis in cases of suspected SM/MCAS (table 3). Published algorithms based on clinical and serological data are available to facilitate a clinical prediction of SM or mMCAS [8].

\section{Evaluation of Tissues in Suspected MCAS}

Evaluation of tissues commonly involved in mastocytosis like bone marrow, skin and mucosa of the gastrointestinal tract is crucial for diagnosis and exact subtyping of MCAS. The routine histological approach focuses on detection of compact mast cell infiltrates in extracutaneous tissues as the main diagnostic criterion for SM. In order not to overlook small mast cell infiltrates, the use of immunohistochemical stains is strongly recommended in all cases of suspected MCAS/SM. Here, tryptase and CD117 (KIT) are the markers of choice for screening tissue sections, allowing even very small mast cell infiltrates to be detected. Proof of expression of CD25 by mast cells demonstrates an aberrant immunophenotype and is defined as a minor diagnostic criterion of SM. Since one major and at least one minor criterion are essential for a diagnosis of SM to be established, detection of small compact mast cell infiltrates with expression of CD25 is sufficient to definitively diagnose SM.

In patients in whom compact mast cell infiltrates are missing, it is of major importance to receive all clinical informations, especially on the presence or absence of skin lesions of urticaria pigmentosa-type, elevated serum tryptase, hematological parameters, and anaphylactic reactions but also on the presence of KIT D816V, which is another minor diagnostic criterion for SM. In this regard, it is noteworthy that the presence of 3 minor SM criteria is also sufficient to establish a diagnosis of SM. Altogether, appropriate staging for SM should include a bone marrow biopsy specimen of at least $2 \mathrm{~cm}$ in length, because of the often very discrete infiltration, with only a few small compact mast cell infiltrates [9]. Moreover, smears of blood and bone marrow must be evaluated to be able to subtype the SM properly. In addition, flow cytometry should be performed if the technique is available. In particular, flow cytometry is helpful in cases where mast cell numbers are low and mast cells do not form compact infiltrates in tissue sections (up to $20 \%$ ) [own unpubl. obs.]. In such patients, flow 
cytometry can still reveal an abnormal mast cell immunophenotype with co-expression of CD25, supporting the diagnosis SM. In a few patients with skin lesions of urticaria pigmentosa-type, staging might reveal absence of SM thus enabling a diagnosis of pure cutaneous mastocytosis ('real' urticaria pigmentosa). In all the cases lacking compact mast cell infiltrates, CD25 expression by mast cells and KIT D816V, mastocytosis can be ruled out definitively. Presence of round and strongly metachromatic, loosely scattered mast cells is best interpreted as mast cell hyperplasia. However, there are no widely accepted criteria for normal or increased numbers of mast cells in various tissue sites [10]. The interpretation of such findings therefore mainly depends on the experience of the pathologist. It should also be stated here that it is impossible to document mast cell activation in tissue sections, unless extensive degranulation with presence of hypogranulated or even degranulated mast cells in large numbers is seen. Finally, it is crucial for the pathologist to be aware of the fact that the evaluation of aberrant marker expression in mast cells may be tricky pending on the organ and tissue sites examined.

Since bone marrow trephine biopsy specimens have been shown to be the main goal for confirmation or exclusion of SM in patients with MCAS a list of the main findings and corresponding diagnoses is given in the following [11]:

(1) Multifocal compact mast cell infiltrates: SM (mainly indolent SM or so-called bone marrow mastocytosis, very rarely aggressive or leukemic SM, compatible with primary MCAS).

(2) Increase in loosely scattered spindle-shaped mast cells with CD25 expression, KIT D816V mutation and chronically elevated serum tryptase but compact tissue infiltrates are lacking: SM (mainly indolent or occult SM but also so-called bone marrow mastocytosis, compatible with primary MCAS).

(3) Increase in loosely scattered spindle-shaped mast cells with KIT D816V +/- CD25 but without elevated serum tryptase: primary monoclonal MCAS.

(4) Increase in loosely scattered round mast cells lacking CD25 and KIT D816V: mast cell hyperplasia (compatible with secondary or idiopathic MCAS).

In contrast to the bone marrow, interpretation of immunohistochemical findings in the gastro-intestinal tract mucosa may be very difficult due to both the often very strong background staining of anti-tryptase and obvious cross-reactivity of cells in the lamina propria mucosae that are definitively no mast cells. Moreover, there are often many CD25-expressing lymphoid and histio-
Table 3. Essential information for the hematopathologist in cases with suspected MCAS or SM*

Typical skin lesions of urticaria pigmentosa KIT mutation status, especially KIT D816V Male sex**

Baseline serum tryptase at the time of biopsy (especially when $>25 \mathrm{~g} / \mathrm{l}^{* *}$ )

Baseline serum tryptase variations over time Additional increase of tryptase during an attack Allergy/anaphylaxy

Presyncopal and/or syncopal episodes**

Serum IgE

Chronic inflammatory disorders

Absence of urticaria and angioedema**

Hepatosplenomegaly

Lymphadenopathy

Ascites and elevated alkaline phosphatase or $\mathrm{LDH}$

Peripheral blood counts

GI tract symptoms

Histamine-related symptoms

Osteoporosis, osteolysis, osteosclerosis

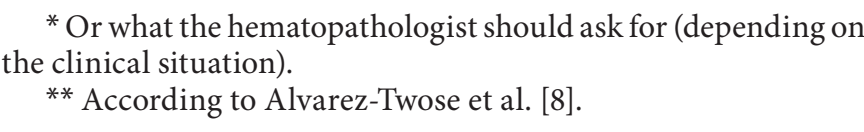

cytic cells making the assessment of CD25 expression by loosely scattered mast cells sometimes very difficult. It should be emphasized here that CD25 immunohistochemistry is easy to interprete in the bone marrow due to the virtual lack of CD25-expressing lymphocytes and histiocytes but clear-cut reactivity of megakaryocytes (serving as internal control) which are easily distinguishable from mast cells. However, finding of an intramucosal spindle-shaped cell with expression of CD25 can be interpreted as atypical mast cell. Of course, in the rare presence of compact often band-like mast cell infiltrates CD25 expression is far more easily to assess (or exclude). The marker of choice for screening of cases of suspected SM/MCAS is anti-CD117 (KIT) which produces a clear staining picture with some loosely scattered mast cells even in normal or inflamed mucosa. The rare occurrence of mast cells in an intra-epithelial position does not allow any conclusions to be drawn about its state, reactive and neoplastic, but is overall more frequently observed in states of marked mast cell hyperplasia and mastocytosis.

In the normal/reactive liver mast cells are virtually absent from the sinusoids but loosely distributed mast cells can be commonly found within portal triads espe- 
cially in chronic inflammation ('hepatitis') or fibrosis/ cirrhosis. Presence of intrasinusoidally located mast cells is highly suggestive of mastocytosis. Expression of CD25 by mast cells is relatively easily assessable in this tissue site. Compact mast cell aggregates are rare and more often observed in the triads than in the sinusoids. Analysis of liver trephine specimens may sometimes be necessary in patients with aggressive or leukemic SM showing marked hepatomegaly but involvement of the liver is, although rarely confirmed, also seen in patients with indolent SM.

Marked splenomegaly and lymphadenopathy are common findings in patients with aggressive or leukemic SM but are rarely present in indolent SM. Thus, in the spleen and in lymph nodes, diagnosis of SM rarely has to be initially confirmed. Due to the high amount of CD25 expression by lymphocytes and histiocytes in both tissue sites difficulties may arise when immunohistochemical findings have to be interpreted to determine an aberrant immunophenotype of mast cells. In the majority of cases, however, multifocal compact mast cell infiltrates enable a diagnosis of tissue involvement by SM to be established at first glance.

Depending on the demonstration or exclusion of mastocytosis and the presence or absence of monoclonal mast cells by appropriate histological immunohistochemical investigation of tissue samples, the following preliminary classification of MCAS is proposed:

(1) Cutaneous mastocytosis with primary MCAS.

(2) Systemic mastocytosis with primary MCAS.

(3) Primary MCAS with KIT-mutated clonal mast cells, but only 2 minor SM criteria: (mono)clonal MCAS (mMCAS).

(4) Secondary and idiopathic MCAS (with and without reactive mast cell hyperplasia or signs of tissue inflammation).
In other words, a primary MCAS can develop in any subtype of mastocytosis and even in the absence of mastocytosis, i.e. when clonal mast cells are detectable but the criteria for SM are not (yet) fulfilled (monoclonal MCAS) [13].

\section{Conclusions}

It is strongly recommended that in all patients with MCAS mastocytosis has to be confirmed or excluded. In cases with cutaneous disease and macroscopically typical efflorescenses a diagnosis of secondary MCAS can be established almost clinically. In all other patients SM has to be proven (or excluded) by histological investigation of a bone marrow biopsy specimen including immunohistochemical studies (tryptase, CD117 and CD25). In addition, molecular analysis should be done in all cases, in order to detect or exclude the presence of the activating point mutation KIT D816V [12]. Other extracutaneous tissues can also be evaluated in this respect, the mucosa of the gastrointestinal tract being of major diagnostic importance. In contrast to the almost standardized approach in the bone marrow diagnosis of mastocytosis with involvement of the mucosa may pose more problems for the pathologist due to several reasons: (1) general pathologists investigating mucosa commonly are less familiar with mastocytosis than hematopathologists; (2) nonspecific staining with antibodies against tryptase and/or CD25 may lead to false-positive diagnoses (which is not the case in the bone marrow due to virtual absence of CD25-expressing lymphoid cells), and (3) compact diagnostic infiltrates are rarely detectable and most diagnoses here have to be based on the presence of three or four minor criteria.

\section{References}

1 Horny HP, Valent P: Diagnosis of mastocytosis: general histopathological aspects, morphological criteria, and immunohistochemical findings. Leuk Res 2001;25:543551.

2 Valent P, Horny HP, Li CY, Longley JB, Metcalfe DD, Parwaresch RM, Bennett JM: Mastocytosis. World Health Organization (WHO) Classification of Tumours. Pathology and genetics; in Jaffe ES, Harris NL, Stein H, Vardiman JW (eds): Tumours of Haema- topoietic and Lymphoid Tissues. Lyon, IARC Press, 2001, pp 291-302.

3 Horny HP, Akin C, Metcalfe DD, Escribano L, Bennett JM, Valent P, Bain BJ: Mastocytosis (mast cell disease). World Health Organization (WHO) Classification of Tumours. Pathology and genetics; in Swerdlow SH, Campo E, Harris NL, Jaffe ES, Pileri SA, Stein H, Thiele J, Vardiman JW (eds): Tumours of Haematopoietic and Lymphoid Tissues. Lyon, IARC Press, 2008, pp 54-63.
4 Akin C, Valent P, Metcalfe DD: Mast cell activation syndrome: proposed diagnostic criteria. J Allergy Clin Immunol 2010;126: 1099-1104.

5 Sonneck K, Florian S, Müllauer L, Wimazal F, Födinger M, Sperr WR, Valent P: Diagnostic and subdiagnostic accumulation of mast cells in the bone marrow of patients with anaphylaxis: monoclonal mast cell activation syndrome. Int Arch Allergy Immunol 2007;142:158-164. 
6 Bonadonna P, Perbellini O, Passalacqua G, Caruso B, Colarossi S, Dal Fior D, Castellani L, Bonetto C, Frattini F, Dama A, Martinelli G, Chilosi M, Senna G, Pizzolo G, Zanotti R: Clonal mast cell disorders in patients with systemic reactions to Hymenoptera stings and increased serum tryptase levels. J Allergy Clin Immunol 2009;123: 680-686.

7 Metcalfe DD, Schwartz LB: Assessing anaphylactic risk? Consider mast cell clonality. J Allergy Clin Immunol 2009;123:687-688.

8 Alvarez-Twose I, González de Olano D, Sánchez-Muñoz L, Matito A, Esteban-López MI, Vega A, Mateo MB, Alonso Díaz de Du- rana MD, de la Hoz B, Del Pozo Gil MD, Caballero T, Rosado A, Sánchez Matas I, Teodósio C, Jara-Acevedo M, Mollejo M, García-Montero A, Orfao A, Escribano L: Clinical, biological, and molecular characteristics of clonal mast cell disorders presenting with systemic mast cell activation symptoms. J Allergy Clin Immunol 2010; 125:1269-1278.

9 Valent P, Akin C, Escribano L, Fodinger M, Hartman K, Metcalfe DD, et al: Standards and standardization in mastocytosis: consensus statement on diagnostics, treatment recommendations and response criteria. Eur J Clin Invest 2007;37:435-453.

10 Valent P, Horny HP, Escribano L, Longley BJ, Li CY, Schwartz LB, Marone G, Nuñez R, Akin C, Sotlar K, Sperr WR, Wolff K, Brunning RD, Parwaresch RM, Austen KF, Lennert K, Metcalfe DD, Vardiman JW, Bennett JM: Diagnostic criteria and classification of mastocytosis: a consensus proposal. Leuk Res 2001;25:603-625.
11 Horny HP, Sotlar K, Valent P: Differential diagnosis of systemic mastocytosis in routinely processed bone marrow biopsy specimens: a review. Pathobiology 2010;77:169-180.

12 Horny HP, Sotlar K, Valent P: Mastocytosis: state of the art. Pathobiology 2007;74:121132.

13 Valent P, Akin C, Arock M, Brockow K, Butterfield JH, Carter MC, Castells M, Escribano L, Hartmann K, Lieberman P, Nedoszytko B, Orfao A, et al: Definitions, criteria and global classification of mast cell disorders with special reference to mast cell activation syndromes: a consensus proposal. Int Arch Allergy Immunol 2012;157:215-225. 